\title{
The Dysplastic Nevus, the True Myth
}

\author{
Ebtisam Elghblawi* \\ MBBCh, MScRes, ADD, DRH, PGC (skin cancer), Private practice (St James Hospitals group), \\ Dermatology OPD, Academic title-associate lecturer at QU MMed skin cancer (Queensland \\ University affiliation) - Australia \\ ebtisamya@yahoo.com
}

\begin{abstract}
The old nomenclature for dysplastic nevi has been modified and changed. Dysplastic nevi are simply one form of melanocytic nevus, and have reached an evolutionary end point and would not develop any further into melanoma. There is no compelling evidence for malignant melanoma transforming at all. Thus each nevus should be looked at differently and avoid over treating.
\end{abstract}

Keywords: Dermoscopy, dysplastic nevi, Melanoma, Nevi.

\section{INTRODUCTION}

Dysplastic nevi (DN) are called the 'funny looking moles'. The misnomer of the word Dysplastic nevi, has just created endless needless fear and anxiety to patients and physicians alike, as well as placing patient lives in jeopardy (Hurwitz RM, and Tavel ME., 2015).

There has been substantial argue about the characterization of dysplastic nevi, the histological and clinical criteria used to label them, and their biological significance, because of the scarcity of transformation of any individual nevus to a melanoma (Goldstein AM, Tucker MA., 2013).

Dysplastic nevus does not only occur in sun-exposed and intermittently sun-exposed areas of the body but also in areas with little or no sun exposure like the scalp, breast, and buttocks (Goldstein AM, Tucker MA., 2013).

A comprehensive literature review conducted by Duffy and Grossman, historically, histologically, clinically and molecularly, concluded that the dysplastic nevus is a type of melanocytic nevus and should be managed like other types of melanocytic nevi and not as melanoma. However the large congenital melanocytic nevi "bathing trunk" mandates careful attention as it carries a considerable risk for melanoma development. Duffy and Grossman believed that such as an expression should be discarded as its misleading and it has become well-established in the new dermatologic language and practice.

DN has been assumed mistakenly long time before to be an intermediate step between nevus and melanoma, where unnecessary over treated and being cut was in the 80s, rendering unsightly cutaneous scars that patient would not forget nor forgive. However there is really no evidence to support the previous claim. It has been found that it is totally benign and not dysplastic at all (Madan R, and Chen S., 2013).

The continued use of the term dysplastic nevus will certainly add to the confusion and controversy of more than 30 years for many more years to come and contribute to the over-treatment of countless patients. Having said that, when a term does not serve the real meaning nor reflect the true nature of a lesion and continues to inflict confusion in the medical field and over treating of patients, then the phrase has to be abandoned and amended. Many propositions had been given, like 'melanocytic nevus' and 'clark nevus' as Ackerman suggested distinguishing between melanocytic nevus (Madan R, Chen S., 2013).

There is considerable debate about the number of DN. Most previous reports agreed that large numbers of DN should be included in the diagnostic criteria. However, that was not agreed (Park KH, Jang YH et al., 2014). 
What is most distressing about the assumption that such dysplasia signifies frank malignancy is that the assumption lacks sufficient objective validation, and is likely erroneous.

Credible academic dermatologists and dermatopathologists have disagreed with the theories surrounding the so-called dysplastic nevus (Hurwitz RM, Tavel ME., 2015).

Medical history is however stuffed with examples of destroyed myths. And medicine across the years had evolved and changed and what was applicable in the past, had been modified and abandoned nowadays (Hurwitz RM, Tavel ME., 2015).

To conclude, the general take is that most DNs are simply a one form of nevus, and have reached an evolutionary end stage and would not develop into melanoma. There is however no incrementing evidence to support the old claim of DNs to transform to malignant melanoma. Thus each nevus should be treated on its merits and the term DN should be abandoned and no need for over treating it and causing the hideous scar.

\section{REFERENCES}

Madan R, Chen S. The so-called dysplastic nevus is not dysplastic at all. Dermatology Practical \& Conceptual. 2013; 3(1):1-2.

Hurwitz RM, Tavel ME. The mythical concept and untoward consequences of a diagnosis of dysplastic nevus: an overdue tribute to A. Bernard Ackerman, MD.Dermatology Practical \& Conceptual. 2015; 5(1):31-34.

Goldstein AM, Tucker MA. Dysplastic Nevi and Melanoma. Cancer epidemiology, biomarkers \& prevention : a publication of the American Association for Cancer Research, cosponsored by the American Society of Preventive Oncology. 2013; 22(4):528-532.

Park KH, Jang YH, Lee WJ, Kim DW, Lee S-J. Four Cases of Dysplastic Nevus Syndrome. Annals of Dermatology. 2014; 26(4):547-549. 